\title{
Design of an active device for controlling lateral stability of fast mobile robot
}

\author{
M. KRID ${ }^{\mathrm{a}}$, F. BENAMAR ${ }^{\mathrm{b}, \mathrm{c}}, \mathrm{Z}$ Z. ZAMZAMI ${ }^{\mathrm{b}}$ \\ mkrid@ksu.edu.sa,amar@isir.upmc.fr,zamzami@isir.upmc.fr \\ ${ }^{a}$ King Saud University, College of Ingineering, Industriel Engineering, Riyadh, KSA. \\ ${ }^{b}$ Sorbonne Universités, UPMC Univ. Paris 06, UMR 7222, Institut des Systèmes \\ Intelligents et Robotiques ISIR, F-75005, Paris, France \\ ${ }^{c}$ CNRS, UMR 7222, ISIR, F-75005, Paris, France
}

\begin{abstract}
Off-road operational conditions require large suspension displacements and a significant clearance between the ground and the main frame, leading to an elevated position of the vehicle mass centre. Consequently, this makes the vehicle more likely to turn over when cornering fast. This paper proposes a new design, and its associated control, for an active device which improves the stability of a fast rover moving up to $8 \mathrm{~m} / \mathrm{s}$.

First, a flexible anti roll bar which couples the right and left robot suspensions is desined. It is used during a steady turning motion and even on an uneven road surface (inclin ed ground). The proposed system increases the controllability of the vehicle by giving access to the roll angle, which is usually uncontrollable.

Then, both rollover dynamic modeling and stability criteria (the Load Lateral Transfer ( $L L T)$ between the right and left wheels) are introduced.

Next, predictive controller based on a dynamic model of the rover is developed to minimizes the the Lateral Load Transfer during cornering and the energy consumed by the actuators of the anti roll active device. The controller and the active anti-roll mechanism were tested using a virtual platform designed using $A D A M S$ dynamic multi-body software, and validated with the SPIDO ROBOT platform. The capabilities of the proposed device
\end{abstract}

Keywords: , Active Anti-Roll System, Roll Dynamics, Vehicle Stability, Lateral Load Transfer Stability Criterion 


\section{Introduction}

Outdoor mobile robots have to perform operations at ever greater distances and ever higher speeds [1][2]. Therefore we are interested in the design and control of fast rovers which are able to move autonomously in a natural environment, following, at high velocity (up to $10 \mathrm{~m} / \mathrm{s}$ ), a virtual corridor referred to by GPS points. Generally, off-road rovers have an elevated centre of gravity for rock collision avoidance, and have an important suspension displacement for obstacle crossing [3]. These two features, combined with a high speed, could cause tip-over situations, generally in the lateral direction. Moreover, current perception systems are not sufficiently accurate or rapid to perform safe autonomous land crossing. In the first case the vehicle can reduce the velocity or/and the steering angle to stabilize the platform [4]. In the second case the velocity of the vehicle is very low [5], it mean that the dynamic of the platfom is not a source of instability. Indeed, the robot could experience major disturbances due to collisions with obstacles or contact failures when crossing holes. These disturbances can create instabilities, or even rover tip-over. Hence, it is interesting to develop active mechanical systems which make system state correction possible when the rover approaches these hazardous situations [6][7][8]. In this context, our work introduces a new solution based on the use of an active anti-roll mechanism combined with a Model Predictive Controller (MPC), which can avoid these situations and increases the stability margin of the vehicle, thus improving the mobility and crossing performance of the robot.

Stability control is a central issue for developing safe navigation on natural terrain. Nowadays, it is done by introducing into the trajectory generator appropriate constraints characterizing the tip-over conditions. [9] considers a stability domain defined in the 2D-space velocity-path curvature and applied to a rigid robot model. [10] considers a complex car model including a tyre relationship to define a predictive control model which optimizes, over a receding horizon, a quadratic criterion composed of the trajectory parameters and the control inputs. This method, and in a similar manner [11][12], utilize wheel torques and/or steering angles as control inputs. A well know example from the field autonromous off-road vehicles is Stanley[13]. Stanford's autonomous vehicle that won the DARPA Grand Challenge race and crossed more than $200 \mathrm{Km}$ in the Mojave desert in almost 7 hours. The vehicle's controller bounded the lateral acceleration to $0.75 \mathrm{~m} / \mathrm{s}^{2}$ to avoid tip-over 
conditions. This stability control approach, limiting system velocity and/or steering angle, can lead to a conservative system, thus reducing system performance. The second approach to vehicle stabilization consists of integrating a dedicated mechanical device. In this paper, we will focus mainly on stabilization in the roll direction and on the minimization of the lateral tip-over risk.

Anti-roll systems have been widely studied and developed in the automotive and railway industries. The main motivation is to increase handling characteristics and decrease rollover accidents. Before the advent of embedded and low cost measurement systems, passive anti-roll bar (or sway bar or stabilizer bar) was the standard technology in most of the vehicles[14]. The passive anti-roll bar is usually in the form of a torsional bar connecting opposite (left / right) wheels together. It generally helps in resisting vehicle body roll motions during fast cornering by increasing the vehicle's roll stiffness. However, the major drawback for the passive linkage is that it introduces a coupling between the suspensions which undesirably transfer the perturbations (bumps, holes, gravel shoulders) from one side to the other. This is called the "copy" effect. In addition, the fixed stiffness of the passive anti roll bar and its ratio between the front and back axles dictate a certain behavior that cannot adapt to the high irregularity of the off-road conditions. Thus, the design of the passive anti-roll bar is subject to conflicting aims.

Historically, the Idea of powered tilt can be dated back to the late 60 s when the British Railways desired to increase the average speed of its fleet in a cost effective way[15]. Therefore, in order to avoid the exorbitant cost of building a new infrastructure that does not exceed the permissible lateral acceleration; the more plausible solution was to modify the train vehicle by providing a mechanical mechanism (air-springs, anti-roll bars, swing bolster, etc...) that compensate for the lateral acceleration by tilting the car body inwards the curve. Nowadays, active tilting is the standard technology for High-speed trains.

In the automotive industry, numerous solutions have already been proposed to equip vehicles with mechatronic solutions including active suspension systems and active stability control systems. Most of these solutions are not suitable for off-road conditions[16] or aim to optimize ride comfort criteria[17] which is of little relevance to the unmanned rover application. However, we mention some of the relevant work done that directly control the roll angle of 
the vehicle. Several approaches, inspired by the passive anti-roll device, use a hydraulic actuator in the place of the torsion bar. This actuator can create an opposite displacement between the left and right suspensions but it does not participate when these displacements are equal (with the same sign). Hydraulic actuation is chosen for its high-force density, robustness, inhibition faculty (bypass mode interconnecting the two chambers of each cylinder) as well as maturity of technology and commercial availability of various parts. BMW developed an Active Roll Stabilization (BMW-ARS) system [18] by installing a hydraulic rotary actuator in the center of the anti roll bar. The roll angle is suppressed by a linear feedback system using the information given by the lateral acceleration sensor. Independent active suspension uses hydraulic struts placed in parallel with the conventional shock absorbers [19] [20] or in series with the spring as in Mercedes Active body control[21]; however, the position-based control of this actuator cannot provide compliant behavior with an adequate time response.[22][23] presented an investigation of an active hydraulic anti-roll bar implemented on heavy articulated vehicle controlled by LQR based method as well as optimal control strategy. It was reported that the designed system reduce the peak normalized load transfer by $20 \%$ in comparison to a passive vehicle.

An alternative approach, proposed by BOSE[24], to replace the conventional passive dampers by linear electromagnetic actuators[25]. Although the system is able to recuperate energy by driving the linear motors in generator mode[26], the high bandwidth active suspensions have high energy demands which make it not suitable for a robotic application with limited energy resources. On the other hand, low-power consumption approaches do not offer direct control of over the roll angle, which lead to degraded performance in high lateral acceleration regime. We mention the concept of passively interconnected suspensions[27] whose principle consists of connecting the hydraulic dampers in various ways by using distributors. The four wheel interconnected systems can provide an increased roll stiffness combined with reduced articulation stiffness and decoupled roll and bounce damping with the expense of increased weight and complexity. A semi-active system, reported in , consisting of a passive anti-roll bar with switchable additional springs, such system can regulate the roll stiffness but cannot directly control the roll angle. [28] implemented Steering and Direct Tilt Control (SDTC) on a narrow three-wheeled tilting vehicle for urban transportation. In the proposed system, the cabin can be directly tilted relative to a non-tilting rear module 
containing the engine and drive system. The controller uses the steer angle and speed to estimate the steady state lateral acceleration and calculates the required tilt angle accordingly. It is however stated that this principle is more effective on narrow vehicles and cannot be easily integrated into off-road vehicles.

This paper presents the design, control and experimental results of an innovative mechatronic device which is able to control the roll stability of the rover in off-road conditions. The proposed system is controlled by a Model Predictive Control (MPC) in realtime, rather than just PID or LQR-based methods. The MPC has the advantage of optimizing current action while anticipating future events and controlling the Active Anti-Roll Bar accordingly. First,we start by presenting the kinematics of the mechanical system and its velocity and force transmission. The next section presents the dynamic model of the vehicle, and especially its roll dynamics and the stability index based on the lateral load transfer. Based on the linearized form of this dynamic model, a model-based predictive control system is designed. This control assumes that the desired path and velocity are known over the receding time horizon. The control optimizes mixed criteria which combine the stability of the robot and its power consumption. Finally, we present simulation results using Matlab-Simulink/Adams co-simulation and the experimental results obtained from a SPIDO ROBOT platform.

\section{Active anti-roll mechanism}

Nowadays, most on-road vehicles are equipped with passive anti-roll bars. Thanks to a flexible bar which couples the right and left suspensions, the roll angle is minimized when the vehicle is cornering. This system acts against any asymmetrical movements between right and left suspensions, especially when the vehicle is submitted to a lateral acceleration (due to centrifugal or gravitational forces). It is used during a steady turning motion and even on an uneven road surface (inclined ground), as shown in Fig.1. The proposed mechanism, inspired by this passive system, replaces the torsion bar by an actuator which, by creating an opposite motion between right and left suspensions, changes the roll configuration of the rover (Fig.2). The horizontal linear actuator, when changing its length, acts on two rocking levers which create two opposite deformations on the right and left double-wishbone suspensions. 

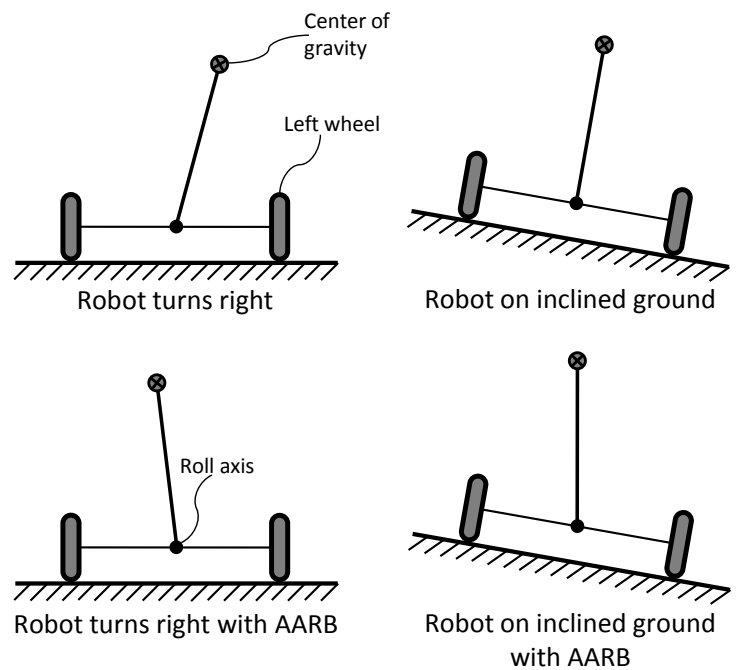

FiguRE 1: Alternative kinematic configuration for the active anti-roll mechanism.

It is clear that this system creates a coupling between the suspensions and can reduce the capacity to cross unilateral discontinuities. However, hydraulic actuation can be rendered almost transparent by interconnecting the two chambers of each cylinder. Thus, by using hydraulic distributors and bypass cylinders, we can defeat this anti-roll system, depending on the ground conditions and other robot parameters.

Figure (3) illustrates another quite similar kinematic configuration which could be used for the conversion mechanism. Contrary to the first configuration, it has symmetrical positions for the rocking lever fixed hinges with respect to the sagittal plane.

Based on dynamic simulations using Adams Software, the velocity and force transmissions appear to be comparable between the two solutions. However, we preferred the first approach, mainly because of the linearity of the transmission characteristic. As can be seen in Figure (4), which illustrates the relationship between the roll angle and the actuator displacement, for the first kinematic configuration the first solution with a horizontal linear actuator exhibits quite linear behaviour in the roll interval of $\pm 15 \mathrm{deg}$. This plot, generated with a periodic sinusoidal motion imposed on the cylinder, seems 


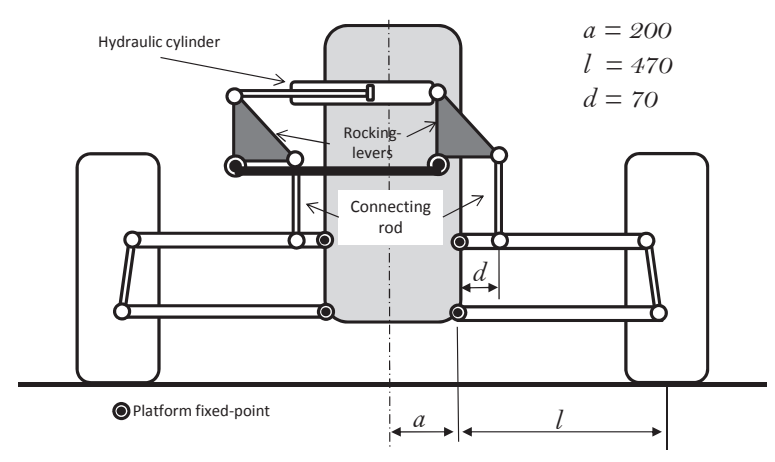

Figure 2: Kinematics of the active anti-roll mechanism.

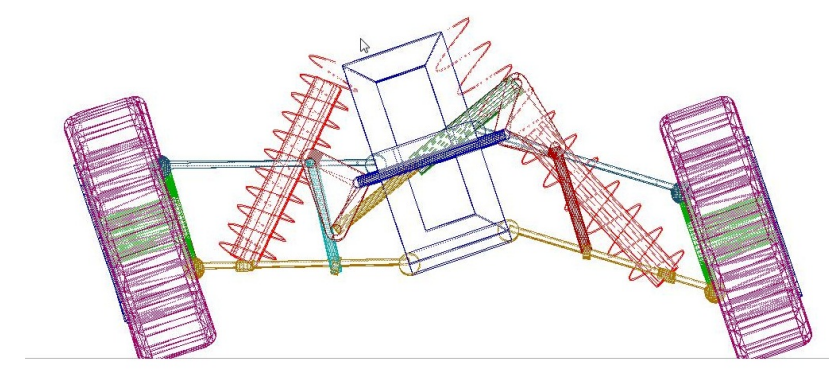

FIgURE 3: Alternative kinematic configuration for the active anti-roll mechanism. 


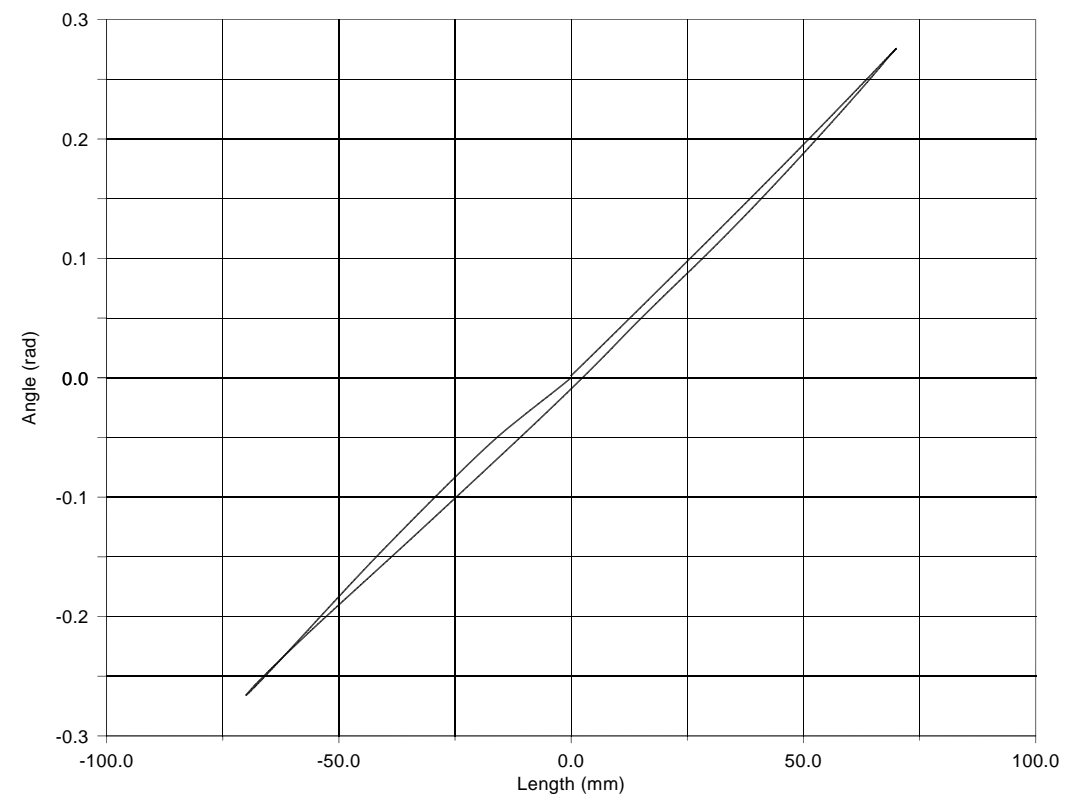

Figure 4: Mechanical characteristics of the first kinematic configurations : horizontal actuator on the left. Relationship between roll angle and linear actuator displacement.

to show some hysteresis. This is due to wheel-ground contact compliance and dynamics effects.

Then mechanical constant defined by the ratio between roll angle change and actuator displacement can be the computed for the reference configuration. Figure (5) shows the location of the Instantaneous Centres of Rotation (ICR) between the mechanism parts, including the ground, chassis, and stub axis, labelled respectively (g), (c) and (s). $I_{i j}$ denotes the ICR of body $i$ with respect to $j$. The stub axis is considered here as being fixed with the wheel, which rotates around the contact centre $I_{s g}$ with respect to the ground. For double wishbone suspensions with parallel links, the roll centre $I_{c g}$ is found to be on the ground plane. By considering $I_{w c}$ and $I_{s w}$, we can define graphically $I_{w g}$, which must be on the intersection of lines $\left(I_{c g}, I_{w c}\right)\left(I_{s g}, I_{s w}\right)$.

Let consider the simplified kinematics of Figure (6) where the stub axle is assumed to be vertical. The velocity with respect to the ground $\left(\vec{v}_{P / g}\right)$ of the attachment point $P$ between the upper wishbone and the transmission vertical link can be decomposed into two components as follows : 


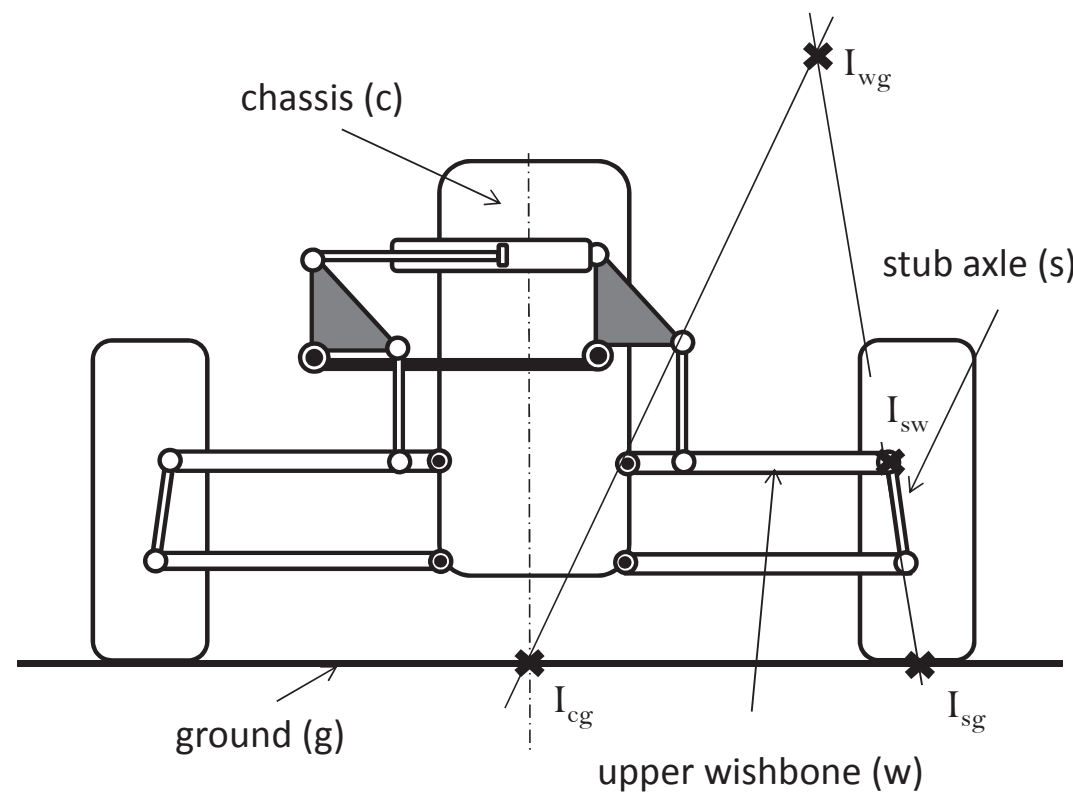

FIGURE 5: Location of the instantaneous centres of rotation.

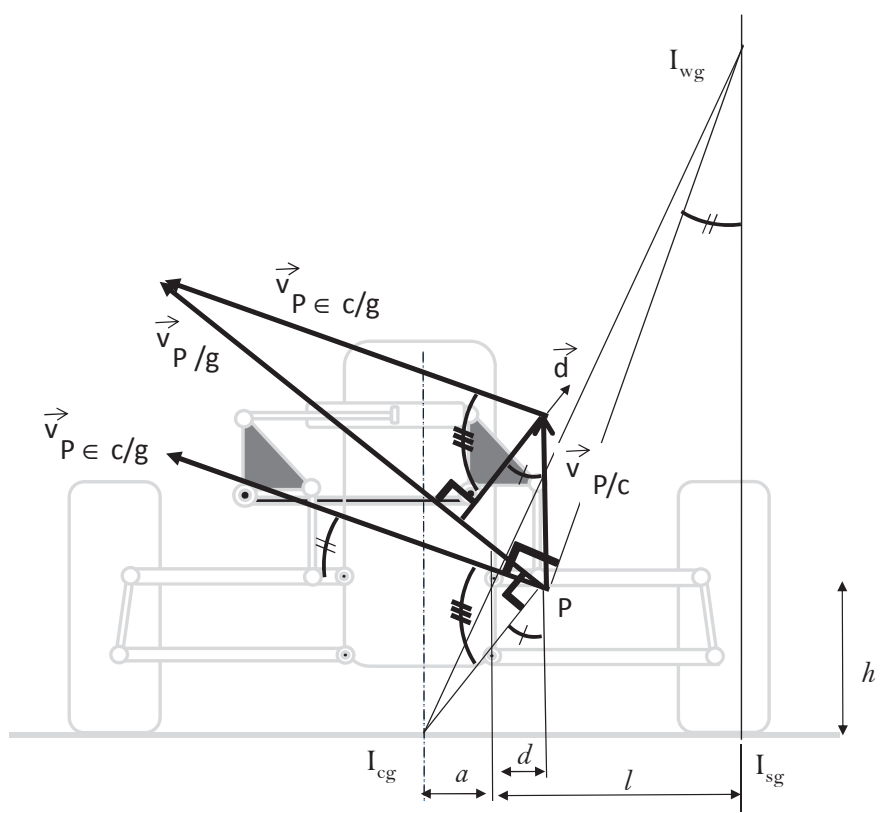

Figure 6: Composition of the linear velocity of point $P$, using the relative velocity formula. 


$$
\vec{v}_{P / g}=\vec{v}_{P / c}+\vec{v}_{P \in c / g}
$$

where $\vec{v}_{P / c}$ is the velocity of $P$ with respect to the chassis, and $\vec{v}_{P \in c / g}$ is the linear component, expressed at point $P$, of the twist describing the motion of the chassis with respect to the ground.

Figure (6) explains how the relationship between the actuator velocity and the roll angular velocity will be found. Let us call $v$ the linear velocity of the actuator. Then, assuming that the rocking levers have the form of isosceles triangles, the velocity w.r.t to chassis of each point $P$ will have a magnitude of $\left|\vec{v}_{P / c}\right|=v / 2$ and will be along the vertical direction. $\vec{v}_{P \in c / g}$ will be orthogonal to the line which connects $P$ to $I_{c g}$, and will have a magnitude of $\left|\overrightarrow{I_{c g} P}\right| \dot{\phi}$, where $\dot{\phi}$ is the roll angular velocity. In addition, $\vec{v}_{P / g}$ is orthogonal to the line $I_{w g} P$.

By projecting the vector equation onto the $\vec{d}$ direction, and by neglecting the distance $d$ relative to $a$ and $l$, we find the following approximate relationship between $v=\frac{d \lambda}{d t}$ and $\dot{\phi}=\frac{d \phi}{d t}$

$$
\frac{v}{\dot{\phi}}=\frac{\delta \lambda}{\delta \phi}=k_{m}=2 d\left(1+\frac{a}{l}\right)
$$

Using the numerical value for $a, d, l$ defined in Figure (2), we find a mechanical constant $k_{m}$ equal to $211 \mathrm{~mm} / \mathrm{rd}$, which could be compared to $195 \mathrm{~mm} / \mathrm{s}$ computed using Adams Software. This ratio will be used in the next section for the dynamic model when expressing the force transmission from actuator to platform roll.

Figure (7) shows the real device mounted on the robot; the hydraulic cylinder and the two rocking levers can easily be identified. Considering power and design constraints, this mechanism is reproduced identically on both front and rear axles.

\section{Vehicle model}

The goal of this section is to develop a vehicle model to design an anti-roll device controller and to provide a measurement for the stability margin. A vehicle is a multi-body system with a complex kinematic architecture having 


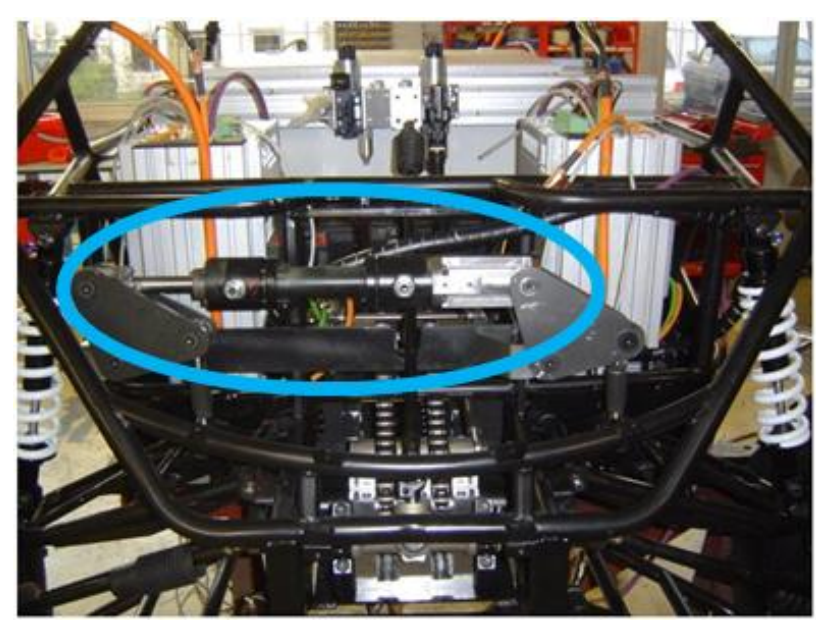

Figure 7: Anti-roll device mounted on the rover.

a complex interaction with the ground. However, the number of degrees of freedom of the model can be reduced to perform a simplified characterization of the vehicle dynamics. The dynamics of each wheel suspension (unsprung mass) will not be considered in this model, but an equivalent 1 DOF model of the suspension will be used to represent the roll motion of the sprung mass [29] [30]. In the model presented in this paper, only the planar dynamic and the roll (Fig.9) are considered. The model is given by [31] :

$$
\begin{aligned}
\dot{V}_{x} M= & M \dot{\psi} V_{y}-M_{s} h_{p} \dot{\psi} \dot{\phi}+\left(F_{x 1}+F_{x 2}\right) \cos \beta_{f}+\left(F_{x 3}+F_{x 4}\right) \cos \beta_{r} \\
& -\left(F_{y 1}+F_{y 2}\right) \sin \beta_{f}-\left(F_{y 3}+F_{y 4}\right) \sin \beta_{r} \\
\dot{V}_{y} M= & M_{s} h_{p} \ddot{\phi}-M \dot{\psi} V_{x}+\left(F_{x 1}+F_{x 2}\right) \sin \beta_{f}+\left(F_{x 3}+F_{x 4}\right) \sin \beta_{r} \\
& +\left(F_{y 1}+F_{y 2}\right) \cos \beta_{f}+\left(F_{y 3}+F_{y 4}\right) \cos \beta_{r} \\
\ddot{\psi} I_{z}= & I_{x z} \ddot{\phi}+a\left(\left(F_{x 1}+F_{x 2}\right) \sin \beta_{f}+\left(F_{y 1}+F_{y 2}\right) \cos \beta_{f}\right) \\
& +d\left(\left(F_{x 2}-F_{x 1}\right) \cos \beta_{f}+\left(F_{y 1}-F_{y 2}\right) \sin \beta_{f}\right) \\
& -b\left(\left(F_{x 3}+F_{x 4}\right) \sin \beta_{r}+\left(F_{y 3}+F_{y 4}\right) \cos \beta_{r}\right) \\
& +d\left(\left(F_{x 4}-F_{x 3}\right) \cos \beta_{r}+\left(F_{y 3}-F_{y 4}\right) \sin \beta_{r}\right) \\
\ddot{\phi} I_{x s}= & M_{s} h_{p}\left(\dot{V}_{y}+\dot{\psi} V_{x}\right)+I_{x z} \ddot{\psi}+M_{s} g h_{p} \phi-2\left(k_{\phi} \phi+c_{\phi} \dot{\phi}\right) \\
I_{\omega} \dot{\omega}_{i}= & C_{m i}-C_{f i}-R_{e} F_{x i}, i=1, \ldots, 4 .
\end{aligned}
$$

In this model (Eq.3), the effect of pitching is not include $\left(\dot{V}_{x}=0\right)$ to reduce the complexity of the vehicle model. The front and rear steering angle $\beta_{f}$ and $\beta_{r}$, the actuator torque on each wheel $C_{m i}$ and the braking 
torque $C_{f i}$ form the input parameters of the navigation control. The side slip angles of the vehicle are introduced in the expression of lateral force $F_{y i}$. Let us define the state variables : $V_{x}, V_{y}$ for the longitudinal and lateral velocities, $V_{\psi}$ for the yaw rate of the vehicle, $\phi$ for the roll angle of the sprung mass and $\omega_{i}$ for the speed of wheel $i$. $M$ depicts the total vehicle mass, $I_{i j}$ are its inertia tensor components, $M_{s}$ the sprung mass, $h_{p}$ the height of the centre of mass with respect to the roll centre (see previous section), $k_{\phi}$ and $c_{\phi}$ are respectively the roll stiffness and damping. These last are related to the stiffness and damping of the spring dampers by linear relationships depending on the geometrical dimensions of the suspension mechanism.

The vehicle dynamics depends on gravity, aerodynamic forces and mainly on ground forces produced by the tyres. The interaction forces between the wheel and the ground are generally described by the semi-empirical Pacejka model [32].If the contact forces are projected on the reference frame of the wheel, as in model (Eq.3), we obtain a non-linear system. The lateral forces $F_{y i}$ and the longitudinal forces $F_{x i}$ appear in all equations of model (Eq.3) except for the fourth term describing the roll dynamics of the sprung mass. The latter will be used to design a control model for the anti-roll system. First, we recall the model of the vehicle without the anti-roll system. If we neglect the term $I_{x z} \ddot{\psi}$ in the roll dynamics equation, we can express the fourth equation of model (Eq.3) as follows :

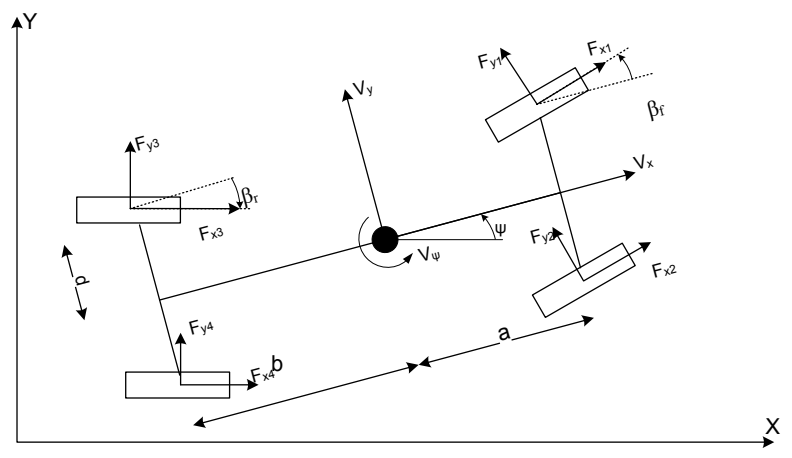

Figure 8: A four-wheeled vehicle with front steering.

$$
\dot{x}_{\phi}=\left(\begin{array}{cc}
0 & 1 \\
a_{21} & a_{22}
\end{array}\right) x_{\phi}+\frac{1}{I_{x s}}\left(\begin{array}{c}
0 \\
M_{s} g h_{p}\left(\dot{V}_{y}+\dot{\psi} V_{x}\right)
\end{array}\right)
$$


with $x_{\phi}=\left(\begin{array}{c}\phi \\ \dot{\phi}\end{array}\right)$ state and $a_{21}=\left(M_{s} g h_{p}-2 k_{\phi}\right) / I_{x s}$ and $a_{22}=-2 c_{\phi}$

The second term of equation (Eq.4) depends on the lateral dynamics and mainly the centrifugal force $M_{s} \dot{\psi} V_{x}$, which is given directly by the vehicle horizontal trajectory. This nonlinear part will determine the equilibrium point of the roll angle associated with the null-control.

If we now consider the anti-roll system introduced in the previous section, clearly it does not change the system parametrization. Thus, we can consider a new torque $u_{\phi}$ due to the cylinder force $F_{a}$ as a new generalized force associated with the roll parameter $\phi$, which will be added to the other terms of equation (Eq.4). Applying the principle of virtual work or kineto-static duality in multibody rigid systems, we can write

$$
u_{\phi}=\frac{\delta \lambda}{\delta \phi} F_{a}=k_{m} F_{a}
$$

where $k_{m}$ is the mechanical constant defined in the previous section. The torque $u_{\phi}$ can be introduced in the fourth equation of the Eq.3. Then the roll dynamic model can be rewritten as follows

$$
\begin{aligned}
& \qquad \dot{x}_{\phi}=A * x_{\phi}+B * u_{\phi}+\frac{1}{I_{x s}}\left(\begin{array}{c}
0 \\
M_{s} g h_{p}\left(\dot{V}_{y}+\dot{\psi} V_{x}\right)
\end{array}\right) \\
& \text { with } A=\left(\begin{array}{cc}
0 & 1 \\
a_{21} & a_{22}
\end{array}\right) \text { and } B=\left(\begin{array}{c}
0 \\
1 / I_{x s}
\end{array}\right) .
\end{aligned}
$$

\section{Controller Design}

The goal of the control of the anti-roll system is the minimization of tipover risks, or the maximization of a stability margin. In our case, the robot cannot control the lateral acceleration because it cannot turn and change direction without undergoing lateral acceleration $\left(a_{y}\right.$ is the consequence resulting from the steering angles and velocity of the robot). With the anti-roll bar, the roll angle can be controlled as shown in the state model (Eq.6) and the centre of gravity position can be changed. To take into account the stability margin in the controller, a stability criterion expression should be formulated to deduce the reference roll angle $\phi_{r e f}$. 


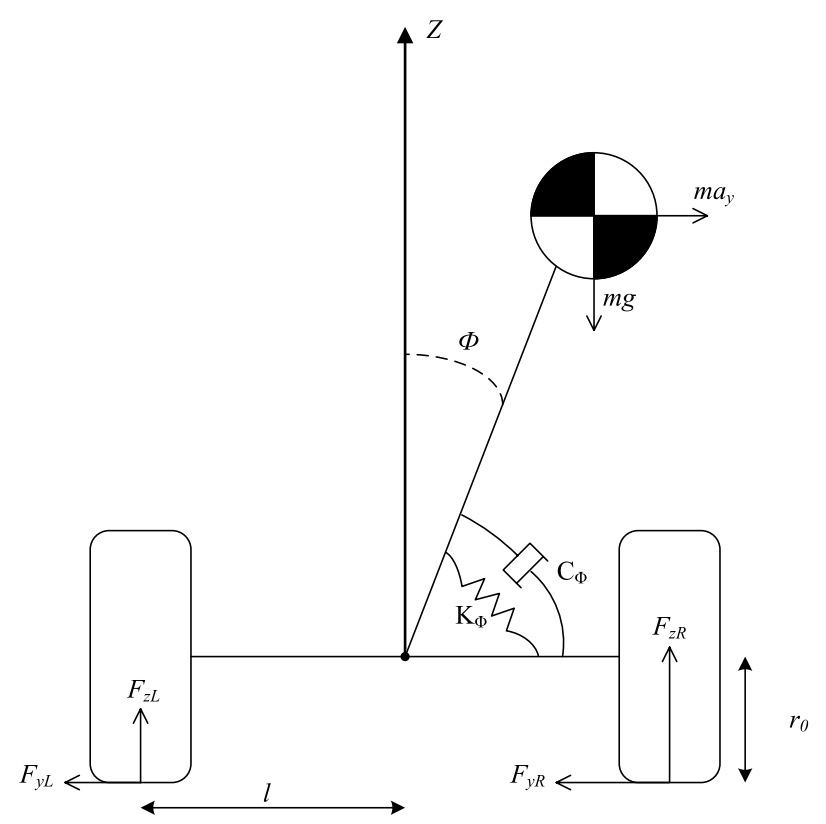

Figure 9: Vehicle model in the roll direction.

\subsection{Load Lateral Transfer}

Numerous stability margins have already been defined, going from a simple static one based on the minimal distance from the mass centre vertical projection to the boundaries of the convex hull support to complex dynamic measurements based on Zero Moment Point $(Z M P)$. However in our dynamic quasi-planar problem, the Load Lateral Transfer $(L L T)$ between the right and left wheels can be used as a measurement of the tip-over risk [33]. Furthermore, the $L L T$ critical threshold is quite easy to tune, since the lift-off of the left or right wheels corresponds to a unitary $L L T$ value. The main objective of the proposed control system will be to stabilize the vehicle by reducing the lateral load transfer $(L L T)$. It is demonstrated in [22] that

$$
L L T=\left|\Delta F_{z}\right|=\left|\frac{M_{s} g h_{p} b}{(a+b) d} \phi+\frac{M h}{d} a_{y}\right|
$$

where $a_{y}$ is the lateral acceleration of the vehicle mass centr and, $h$ is its height with respect to the ground surface. Solving the equation $L L T=0$, we obtain a reference roll angle $\phi_{\text {ref }}$ : 


$$
\phi_{\text {ref }}=-\frac{M h(a+b)}{M_{s} g h_{p} b} a_{y}(t)
$$

which minimizes the lateral load transfer (Eq.7).

As can be seen in expression of Eq.8, $\phi_{\text {ref }}$ depends on the lateral acceleration $a_{y}(t)$. Thus it is necessary to replace the lateral acceleration $a_{y}(t)$ by $a_{y_{\text {ref }}}$. This latter can be computed easily from the reference robot trajectory. In our case the reference path and the desired speed are assumed to be known at this control level. If we neglect the side slip angle of the vehicle mass centre, we can determine $a_{y_{r e f}}$ from the path curvature $\kappa_{r e f}(t)$ and the forward velocity $V_{x r e f}(t)$ though the following relationship

$$
a_{y_{r e f}}(t)=-k_{r e f}(t) V_{x r e f}^{2}
$$

Then, using the expression of $a_{y_{r e f}}$ in equation Eq.9, equation (Eq.8) becomes

$$
\phi_{r e f}(t)=\frac{M h(a+b)}{M_{s} g h_{p} b} k_{r e f}(t) V_{x_{r e f}}^{2}
$$

This reference angle is bounded to the extremal values of the roll angle, determined directly by the cylinder stroke. In order to take into account the dynamic constraints and to anticipate future set point changes, a modelbased predictive control $(M P C)$ method is used. We start by splitting the control input into two terms, i.e.

$$
u_{\phi}=u_{\phi l}+u_{\phi c}
$$

where

$$
u_{\phi l}=-M_{s} g h_{p}\left(\dot{V}_{y}+\dot{\psi} V_{x}\right)
$$

is a linear decoupling term which can be estimated from the robot on-board sensors. Thus the non-linear model (Eq.4) now takes the following linear form :

$$
\dot{x}_{\phi}=\left(\begin{array}{cc}
0 & 1 \\
a_{21} & a_{22}
\end{array}\right) x_{\phi}+\left(\begin{array}{c}
0 \\
1 / I_{x s}
\end{array}\right) u_{\phi c}
$$


The continuous-time state space model (Eq.13) can be discretized, assuming the zero-order-hold method. We denote by $T_{d}$ the timesteps and assume that the state matrix is nonsingular (juste verify that $a_{21} \neq 0$ ). It is easy to calculate an approximate discrete model, based on that for small timesteps. The approximate solution then becomes :

$$
\begin{aligned}
x_{\phi}(k+1) & \approx\left(I+A * T_{d}\right) x_{\phi}(k)+T * B u_{\phi c}(k) \\
y(k) & =C x_{\phi}(k)
\end{aligned}
$$

The equation (Eq.14) can be transformed into the following discrete state space model :

$$
\begin{aligned}
x_{\phi}(k+1) & =A_{k} x_{\phi}(k)+B_{k} u_{\phi c}(k) \\
y(k) & =C_{k} x_{\phi}(k)
\end{aligned}
$$

where $A_{k}=I+A * T_{d}$ and $B_{k}=T * B$ are the state space matrix and $C_{k}=\left(\begin{array}{ll}1 & 0\end{array}\right)$.

\subsection{Control System}

Fig.10 shows a block diagram of the proposed predictive control $M P C$ approach. In this section, the goal for the predictive controller block is to minimize the objective function. Before that, we must estimate the state of the system in the Dynamic Model block.

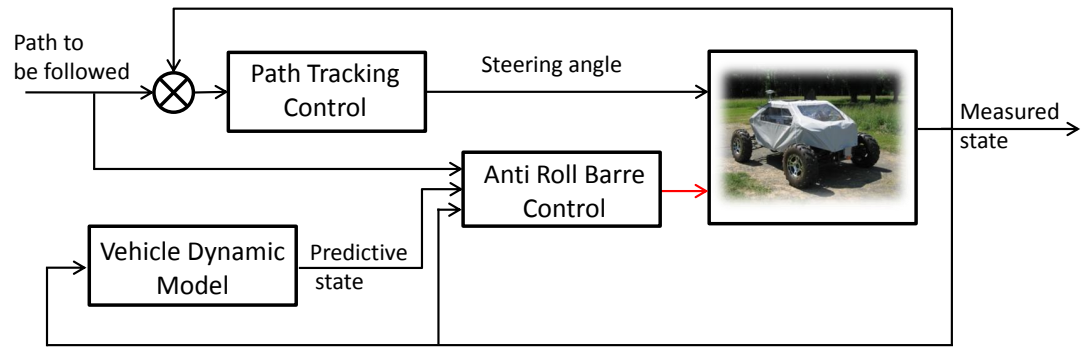

FiguRE 10: Block diagram for the proposed model predictive controller.

In order to take into account the estimated state of our robot in the formulation of controller, it must be written with respect to the input and the state. $\hat{y}_{t+1, t}$ can be described as follows : 


$$
\begin{aligned}
\hat{y}_{t+1, t} & =C x(t+1) \\
& =C_{k}\left(A_{k} x(t)+B_{k} u(t)\right) \\
& =C_{k} A_{k} x(t)+\left(C_{k} B_{k}\right) u(t) \\
& =C_{k} A_{k} x(t)+\left(C_{k} B_{k}\right) u(t-1) \\
& +\left(C_{k} B_{k}\right) \Delta u_{t, t}
\end{aligned}
$$

Then we can deduce $\hat{y}_{i+1, t}$, depending on $x(t), u(t-1)$ and $\Delta u_{i, t}$ Let consider at instant $t$ the objective function $\mathrm{J}$ defined by :

$$
J\left(x_{\phi}(t), \Delta U(t)\right)=\sum_{i=t+1}^{t+p}\left\|\phi_{i, t}-\phi_{r e f_{i, t}}\right\|_{Q}^{2}+\sum_{i=t}^{t+c-1}\left\|\Delta u_{i, t}\right\|_{R}^{2}
$$

which represents a weighted sum of the reference tracking errors of $\phi_{i, t}$ (the predicted value of $\phi$ at time $i$ computed at instant $t$ ) and of control input changes $\Delta U(t)=\left[\Delta u_{i, t}, \ldots, \Delta u_{t+c-1, t}\right]$. These latter components are defined by

$$
u_{i, t}=u_{i-1, t}+\Delta u_{i, t}
$$

$Q$ and $R$ are weighting matrices and $p$ and $c(c \leq p)$ are the prediction time horizon and control time horizon, respectively. After the resolution of the quadratic optimization problem (Eq.16), only the first term of $\Delta U(t)$ is used to compute the control inputs $u_{\phi c, t}$ of the linearized model that must be applied to the system, that is to say

$$
u_{\phi c, t}=u_{\phi c, t-1}+\Delta u_{t, t}
$$

The prediction controller calculates $\Delta u_{i, t}$ by minimizing the quadratic criterion (Eq.16).

$$
\frac{\partial J}{\partial \Delta u}=0
$$

This roll controller can be considered as being decoupled from the one dedicated to path tracking. The path controller unit manages trajectory tracking by adjusting the speed and the steering angles that minimize the error between the reference trajectory and the actual position of the robot. The roll controller acts directly on the anti-roll actuator to enhance vehicle stability by minimizing the roll angle and the lateral load transfer $(L L T)$. 


\section{Results}

The $M P C$ controller and the active anti-roll mechanism presented in the previous section were tested. In this section, various results are presented. First, advanced simulation results, obtained from a virtual platform designed using Adams dynamic multi-body software as shown in Figure(11), are reported. The second part of this section presents the experimental results, recorded with the SPIDO ROBOT platform shown in Figure(12).

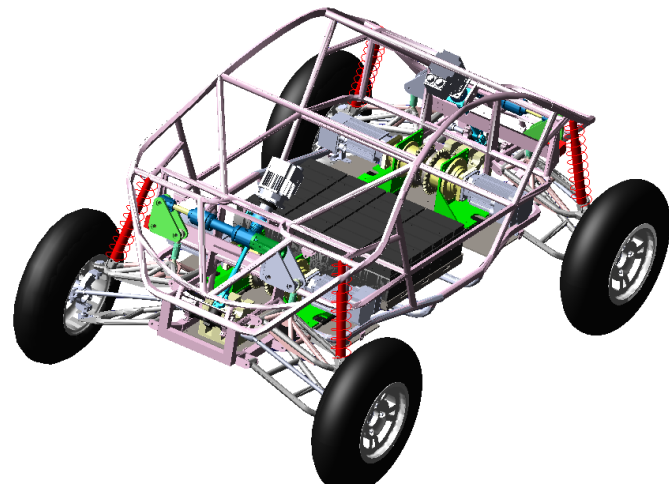

Figure 11: Adams virtual platform.

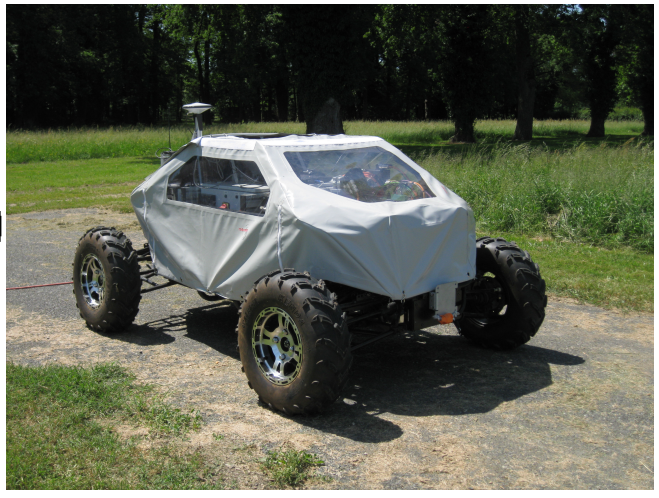

FigurE 12: Experimental platform.

\subsection{Simulation results}

We begin the controller validation by using Matlab-Simulink/ADAMS co-simulation. Adams software computes the vehicle multi body dynamics, which includes a wheel-ground contact model. The Adams model is a fourwheeled chassis with independent double-wishbone suspensions and with 2 anti-roll mechanisms integrated on each axle (Fig.11). The contact model includes wheel stiffness and damping and a regularized Coulomb friction law parameterized by static and dynamic friction coefficients. Matlab-Simulink recovers the Adams output (i.e. the roll angle), computes the optimal control input (i.e. the actuator force) and sends it back to Adams.

The goal of the simulation tests is to quantify the benefits of the anti-roll device, especially when the vehicle performs a cornering manoeuvre. First, we will present simulation results for a circular path obtained by constant control inputs : $\beta_{f}=12$ for the front steering angle and $V_{x}=5 \mathrm{~m} / \mathrm{s}$ for the forward velocity. The circular path starts from instant $t=3 \mathrm{~s}$, and is preceded by a straight-line path for the acceleration phase. 


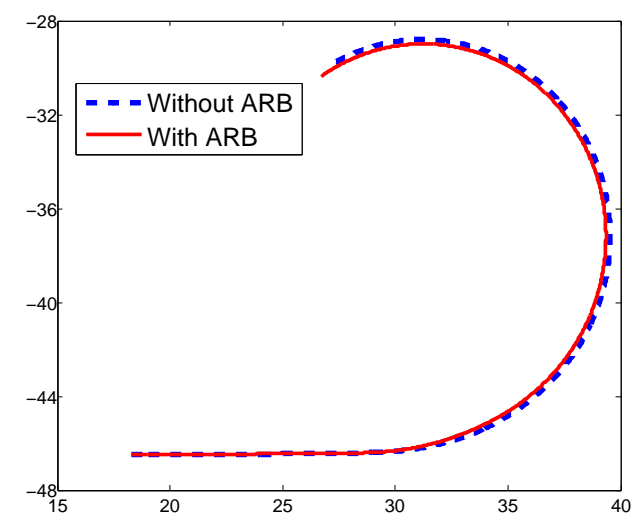

Figure 13: Robot centre path.

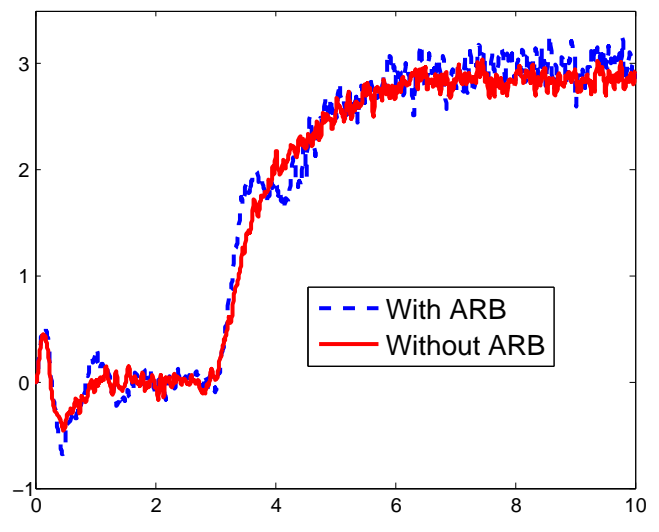

FiguRE 14: Lateral acceleration with respect to time.

The curves of Figure (13) and (14) show the robot trajectories and lateral accelerations in both cases, with and without the anti-roll device. By comparing these curves, we can conclude that it is possible to neglect the anti-roll effect on the horizontal robot motion and the availability of path/posture decoupling. This enables us to integrate the anti-roll device without changing the path control signals.

In Figure (15), we show the Normalized Lateral Load Transfer NLLT with respect to time for both cases : with and without the anti-roll control. Normalization is done by dividing $L L T$ by the total weight. Thanks to the anti-roll control, the load transfer is minimized : NLLT $\approx 0.1$, compared to the passive case where $N L L T=0.45$. Curve oscillations are mainly due to the high eigenfrequency introduced by the contact stiffness of the Adams contact model.

For this test, we also present in Figure (16) the roll angle and the control input, i.e. the actuator force. We can see that the roll control provides a roll behaviour which is contrary to the natural behaviour (without control). This behaviour resembles that of motorcycle racer who leans into the turn for centrifugal force compensation.

The benefits of the active anti-roll system can be further demonstrated when increasing the velocity displacement $V_{x}$. Figure (17) shows the lateral load transfer as a function of the vehicle speed and with a constant steering angle. By comparing the vehicle's LLT with and without the active roll device, we can clearly notice the benefits of this system as regards tip-over stability. 
For the uncontrolled roll case, the robot exhibits instability when the speed reaches $7 \mathrm{~m} / \mathrm{s}$; in this case the $N L L T$ exceeds the limit of 1 . However, the anti-roll control increases the stability margin of the robot, as the $L L T$ is minimized, opening the possibility for the robot to travel at a speed of greater than $7 \mathrm{~m} / \mathrm{s}$ with a sufficient safety margin ( $N L L T$ remains below 0.5$)$.

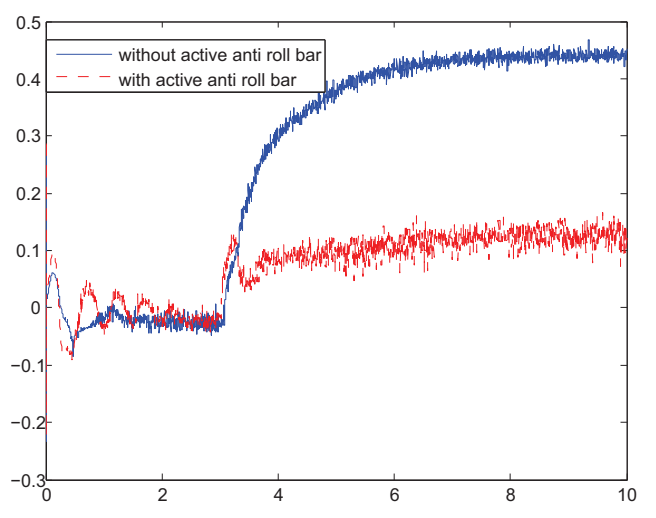

Figure 15: $L L T$ with respect to time.

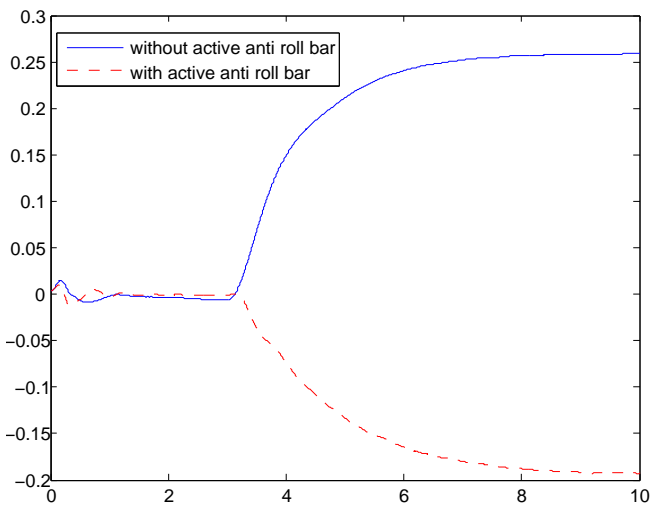

FiguRE 16: Roll angle.

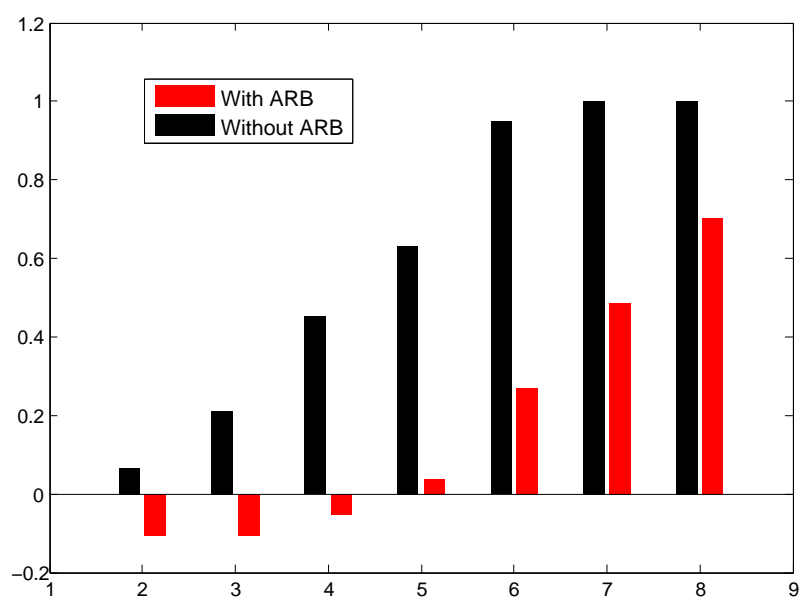

Figure 17: Normalized Lateral Load Transfer as a function of robot speed.

\subsection{Experimental results}

The experimental platform is an all-terrain four-wheel-steering vehicle, as shown in Figure(12). The vehicle weight is $420 \mathrm{~kg}$. The front and rear half 
wheelbases are $0.62 m$ and $0.58 m$, respectively. It is equipped with a Real Time Kinematic GPS (RTK-GPS) which provides a localization with respect to a reference station which is accurate to within $\pm 2 \mathrm{~cm}$. The active anti roll bar $(A A R B)$ is easily integrated into the $U G V$, since it only requires an adequate hydraulic power system, with minor modifications to the structural design of the $U G V$.

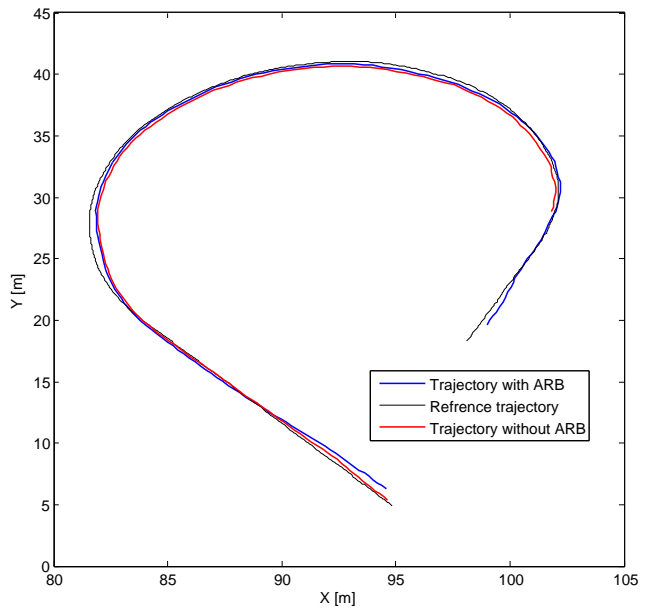

FiguRE 18: Trajectory at $5 \mathrm{~m} / \mathrm{s}$.

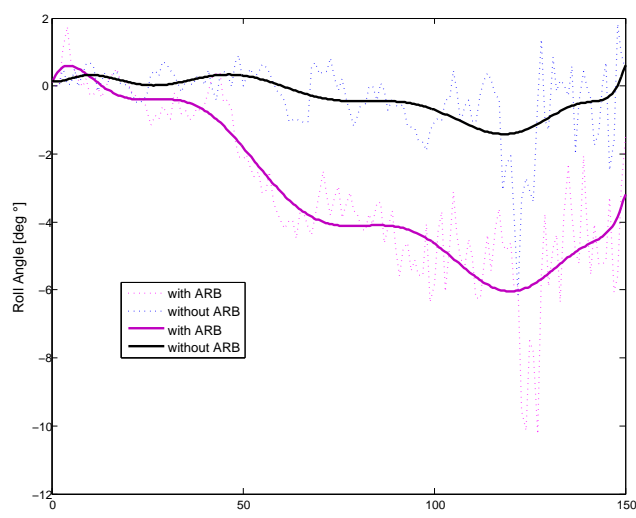

Figure 20: Roll angle at $5 \mathrm{~m} / \mathrm{s}$ as a function of time.

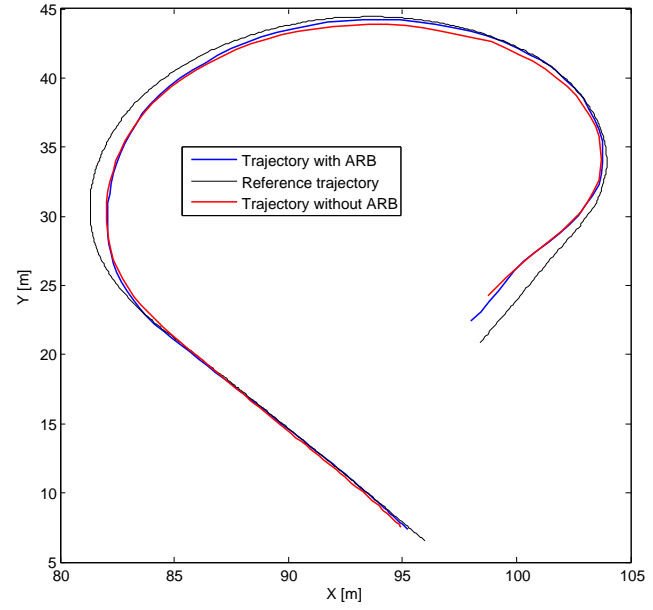

FiguRE 19: Trajectory at $8 \mathrm{~m} / \mathrm{s}$.

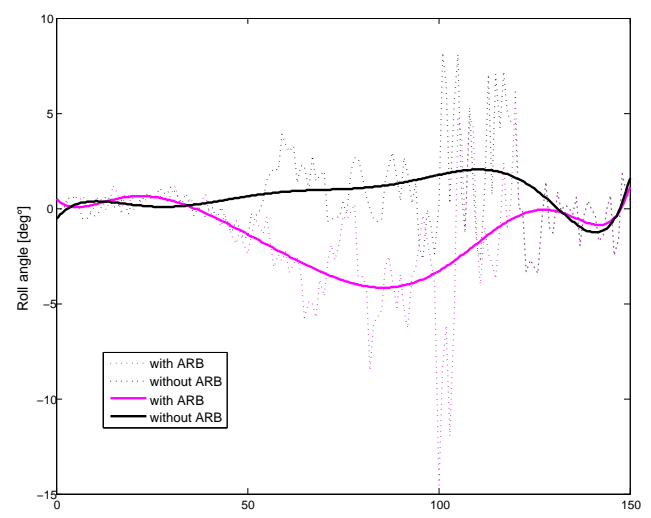

Figure 21: Roll angle at $8 \mathrm{~m} / \mathrm{s}$ as a function of time. 


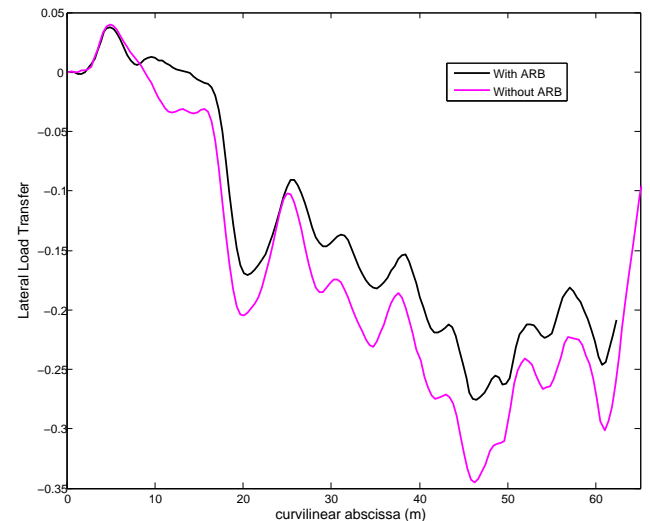

Figure 22: Normalized Lateral Load Transfer $L L T$ at $5 \mathrm{~m} / \mathrm{s}$.

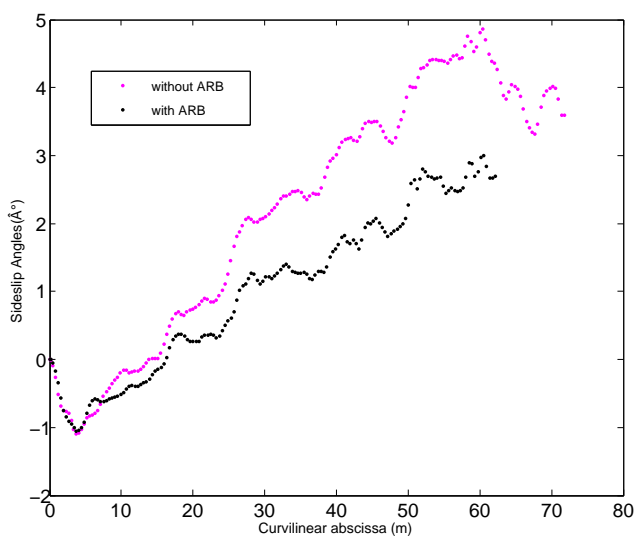

Figure 24: Slip angle at $5 \mathrm{~m} / \mathrm{s}$.

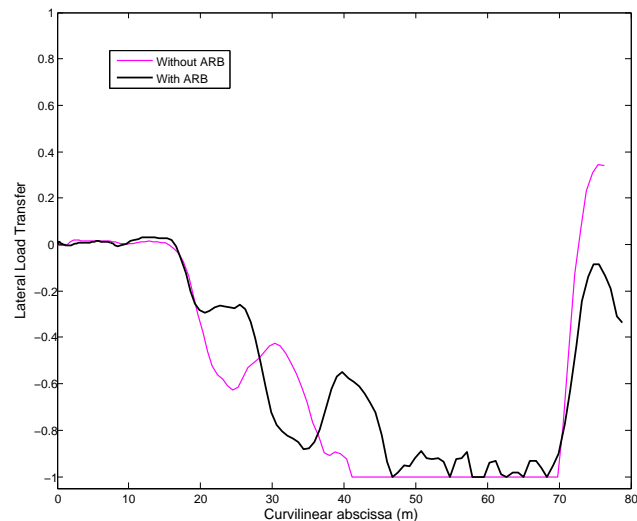

Figure 23: Normalized Lateral Load Transfer $L L T$ at $8 \mathrm{~m} / \mathrm{s}$.

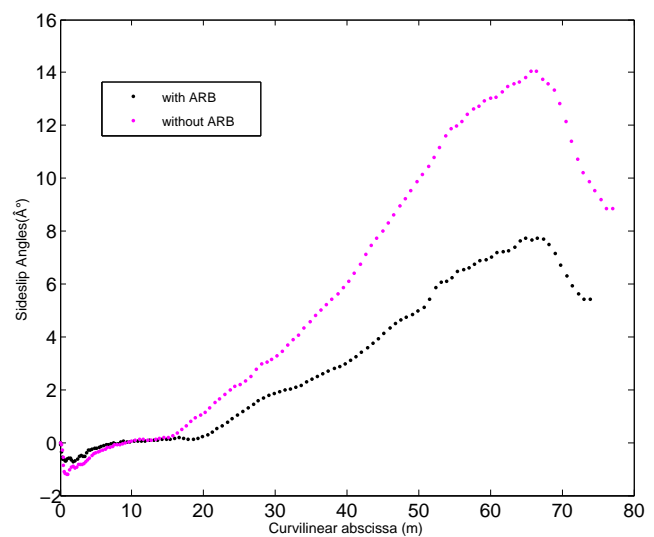

FiguRE 25: Slip angle at $8 \mathrm{~m} / \mathrm{s}$.

An analysis of the influence of the $A A R B$ on the overall performance of the $U G V$ is performed by first studying its impact on the trajectory tracking task. A comparison is made between two identical experimental trial scenarios with the same trajectory tracking control, with the exception that in one case the $A A R B$ is activated while in the other it is disabled or rendered transparent as discussed earlier. Figures (18) and (19) show the trajectory of the centre of mass of the robot at different speeds. Observation of the results shows that the trajectory of the robot is not disturbed by the $A A R B$. In Figures (18) and (19) the two trajectories for the $U G V$ with and without 
the $A A R B$ are almost superimposed, though the activation of the $A A R B$ enables a better tracking of the reference trajectory. The UGV's trajectory at $5 \mathrm{~m} / \mathrm{s}$ and $8 \mathrm{~m} / \mathrm{s}$ is slightly better when the $A A R B$ is enabled, as shown in figures (18) and (19). This slight difference becomes visible when cornering, because the roll angle of the $U G V$ is not the same as that shown in figures (20) and (21).

Initial observations show that by activating the $A A R B$, the $U G V$ can tilt towards the interior of the curve, hence increasing roll stability. On the other hand, when the $A A R B$ is deactivated the $U G V$ tilts outwards during turning, as shown in figures (20) and (21), which progressively increases the roll-over risk. Figures (20) and (21) show a relatively small roll angle at $5 \mathrm{~m} / \mathrm{s}$, but a significant roll angle is observed at $8 \mathrm{~m} / \mathrm{s}$, depicted by the blue line. The activation of the anti roll bar has a direct impact on the roll angle. Moreover, the centre of mass of the $U G V$ moves from the left (the outside of the corner), represented by the blue line in the figure, to the right (the inside of the corner), represented by the red line, with and without the $A A R B$, respectively.

This balancing of the roll angle of the frame of the robot directly generates a variation in the lateral load transfer. Figures (22) and (23) show a clear difference in the vehicle state between the activated and deactivated modes of the $A A R B$. From the observation of the variations in the value of the lateral load transfer at $5 \mathrm{~m} / \mathrm{s}$ we can deduce that the absolute value of the $L L T$ defined in (7) is less when the $A A R B$ is active (shown in red) than when the $A A R B$ is deactivated (blue). Once the speed reaches $8 \mathrm{~m} / \mathrm{s}$ while cornering, the stability limit is reached as the $|L L T|=1$. This limite of stability or unstability is detected from the curvilinear abscissa $40 \mathrm{~m}$ to $70 \mathrm{~m}$ as show in figure 23. We can deduce that the robot is unstable from the beginning of the turn when it start changing direction without the $A A R B$. The instability signify that the robot has no cantact with the ground on one wheel or more $(|L L T|=1)$. In this situation a significant difference is observed in the vehicle's performance between the use of the $A A R B$ and the deactivated case. This mainly due to the fact that without the $A A R B$ the $U G V$ reaches the stability limit at $41 \mathrm{~m}$ on the curvilinear abscissa, while by employing the $A A R B$ the stability limit is reached at $47 m$, making a 6 -meter difference. Moreover, peaks are observed on the LLT curves, representing the instants where the $U G V$ loses contact with the ground. This is a phase where the robot is no longer controllable. These extreme situations disappear when the $A A R B$ is activated, hence increasing stability since the $U G V$ stays 
within the stability limit zone of $L L T=1$ while remaining in contact with the ground for the 4 wheels.

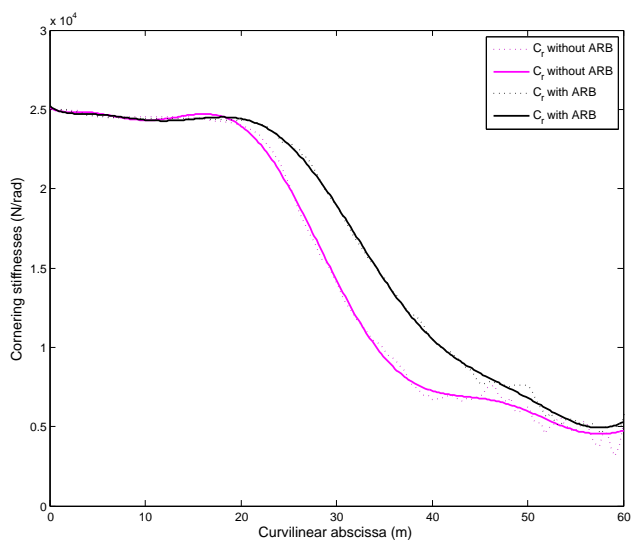

Figure 26: Cornering stiffnesses at the rear of the robot at $5 \mathrm{~m} / \mathrm{s}$.

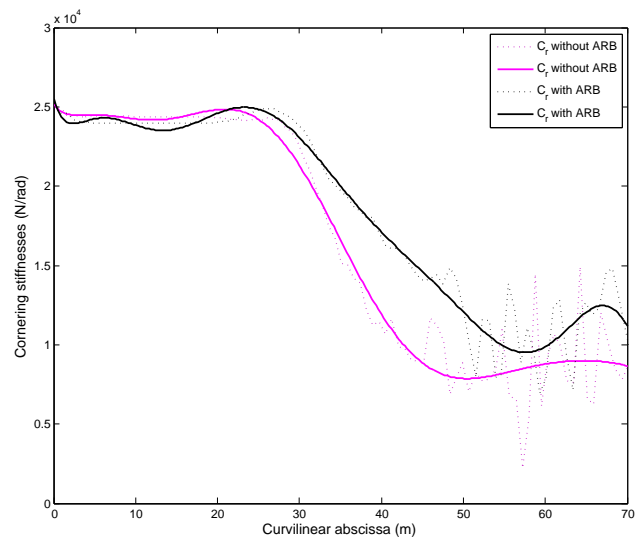

Figure 28: Cornering stiffnesses at the rear of the robot at $8 \mathrm{~m} / \mathrm{s}$.

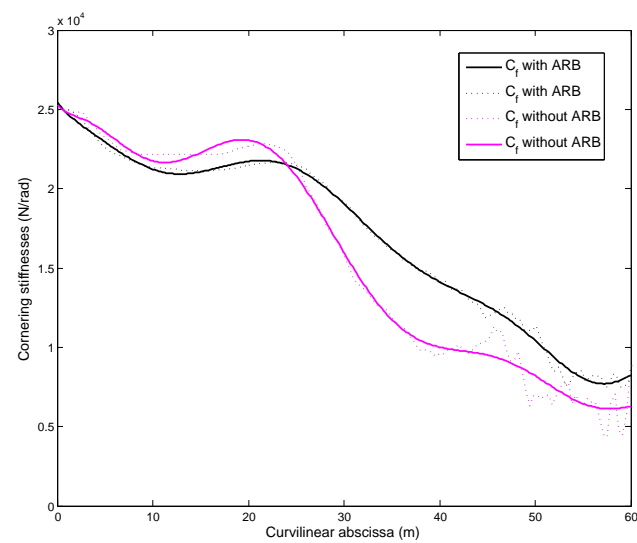

Figure 27: Cornering stiffnesses at the front of the robot at $5 \mathrm{~m} / \mathrm{s}$.

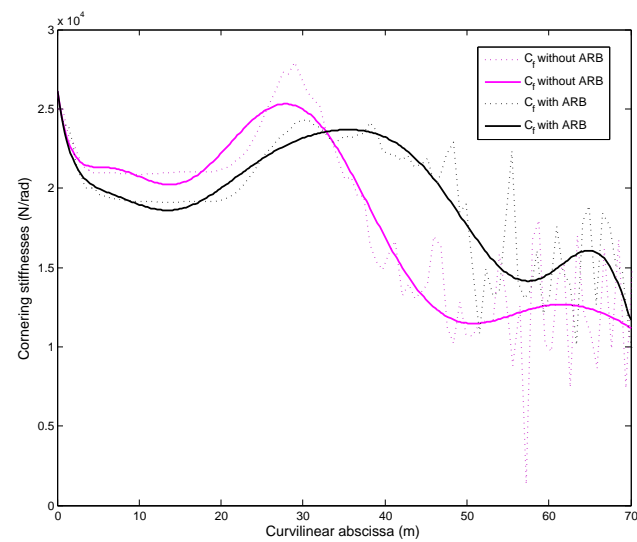

Figure 29: Cornering stiffnesses at the front of the robot at $8 \mathrm{~m} / \mathrm{s}$.

Finally, we can conclude this analysis by observing the slip angle. This variable characterizes a phenomenon that is essential in changing the direction the vehicle and which quickly turns into a source of instability when it exceeds certain limits. In our analysis of the experimental results we noticed a slight difference in the trace of the slip angle between the cases of an 
activated and deactivated $A A R B$. This difference is larger when the speed increased (figures (24) and (25)).

This observation is confirmed by the cornering stiffness trace. It can be seen that the variation in $C_{f}$ as shown in fig.27 and fig.29, and $C_{r}$ in fig.26 and fig.28, is less marked when the anti roll bar is activated. This variables are deduced from the previous work of Lenin et al. in [34]. The cornering stiffness and the sideslip angles are estimated online with a kinematic and dynamic observer respectively. Based on the Pacejka study of the variation in the curve slope $F_{y}=f(\alpha)$ according to the vertical load $F_{z}$ in [32], we can deduce that the minimisation of the lateral load transfer on a bend or on inclined ground generates a minimisation of cornering stiffness variation, and therefore reduces sliding on the whole robot. This provides us with the perspective of studying the effect of the anti-roll bar on the stability of the robot.

\section{6. conclusion}

In this paper an integrated approach to designing an active anti-roll system was presented. An innovative kinematics scheme, which can easily be added to an existing off-road chassis, is proposed. It can replace the standard anti-roll bar used on most vehicles. The input $u_{\phi}$ improves the controllability of the lateral dynamics of the vehicle and makes it possible to control the roll angle. The reference roll angle was generated by maximizing the LLT stability margin. In order to take into account the discontinuity and the variations in input, a predictive controller model based on the minimization of load transfer and energy consumption was designed. Simulation results show that this system improves the performance and stability of the robot when cornering. The experimental results, recorded using the SPIDO ROBOT platform, confirm the simulation results. Moreover, the proposed system allows the robot to increase its velocity in the turn. An important advantage of the proposed solution is its easy integration as a replacement part, without any transformation of the original chassis. This system can be controlled independently, and is demonstrated to have no effect on the dynamics of the path controller.

\section{Références}

[1] M. Richier, R. Lenain, B. Thuilot, C. Debain, Dual back-stepping observer to anticipate the rollover risk in under/over-steering situations. 
application to atvs in off-road context, in : IEEE/RSJ International Conference Intelligent Robots and Systems, IROS 2012, pp. 5387-5393.

[2] E. Lucet, C. Grand, D. Salle, P. Bidaud, Stabilization algorithm for a high speed car-like robot achieving steering maneuver, in : IEEE International Conference Robotics and Automation, ICRA 2008, pp. 2540-2545.

[3] N. Bouton, R. Lenain, B. Thuilot, P. Martinet, An active anti-rollover device based on predictive functional control : application to an allterrain vehicle, in : IEEE International Conference Robotics and Automation, ICRA 2009, pp. 1309-1314.

[4] M Richier, R Lenain, B Thuilot, C Debain, Rollover Prevention System Dedicated to ATVs on Natural Ground, Applied Mechanics and Materials, 2012, 162-505.

[5] Faiz Benamar and Christophe Grand, Quasi-Static Motion Simulation and Slip Prediction of Articulated Planetary Rovers Using a Kinematic Approach, J. Mechanisms Robotics 5(2), 021002 (Mar 26, 2013) (13 pages)

[6] N. Bouton, R. Lenain, B. Thuilot, P. Martinet, A new device dedicated to autonomous mobile robot dynamic stability : Application to an offroad mobile robot, in : IEEE International Conference Robotics and Automation, ICRA 2010, pp. 3813-3818.

[7] P. Gaspar, Z. Szabo, J. Bokor, The design of an integrated control system in heavy vehicles based on an lpv method, in : 44th European Control Conference Decision and Control, 2005, pp. 6722-6727.

[8] A. Diaz-Calderon, A. Kelly, Development of a terrain adaptive stability prediction system for mass articulating mobile robots, in : S. Yuta, H. Asama, E. Prassler, T. Tsubouchi, S. Thrun (Eds.), Field and Service Robotics, Vol. 24 of Springer Tracts in Advanced Robotics, Springer Berlin Heidelberg, 2006, pp. 343-354.

[9] M. Spenko, Y. Kuroda, S. Dubowsky, I. K., Hazard avoidance for highspeed mobile robots in rough terrain, Journal of Field Robotics 23 (5) 2006, pp. 311-331. 
[10] G. Palmieri, P. Falcone, H. Tseng, L. Glielmo, A preliminary study on the effects of roll dynamics in predictive vehicle stability control, in : 47th IEEE Conference Decision and Control, CDC 2008, pp. 5354-5359.

[11] C. Zhao, W. Xiang, P. Richardson, Vehicle lateral control and yaw stability control through differential braking, in : Industrial Electronics, 2006 IEEE International Symposium on, Vol. 1, pp. 384-389.

[12] Y. Seo, J. Choi, G. Duan, Lateral vehicle control using the ccv mode control, in : Control Conference, CCC 2006, Chinese, pp. 41-46.

[13] S. Thrun, M. Montemerlo, H. Dahlkamp, D. Stavens, A. Aron, J. Diebel, P. Fong, J. Gale, M. Halpenny, G. Hoffmann, K. Lau, C. Oakley, M. Palatucci, V. Pratt, P. Stang, S. Strohband C. Dupont, L.-E. Jendrossek, C. Koelen, C. Markey, C. Rummel, J. van Niekerk, E. Jensen P. Alessandrini, G. Bradski, B. Davies, S. Ettinger, A. Kaehler, A. Nefian, and P. Mahoney Winning the darpa grand challenge. Journal of Field Robotics, 2006.

[14] Dieter Schramm, Manfred Hiller, and Roberto Bardini. Force components. In Vehicle Dynamics, pages 216-219. Springer Berlin Heidelberg, 2014.

[15] AC Zolotas. Advanced control strategies for tilting trains. Phd, Loughborough University, 2002.

[16] J. Gohl, R. Rajamani, L. Alexander, and P. Starr. Active roll mode control implementation on a narrow tilting vehicle. Vehicle System Dynamics, 42(5) :347-372, 2004.

[17] P.S. Els, N.J. Theron, P.E. Uys, and M.J. Thoresson. The ride comfort vs. handling compromise for off-road vehicles. Journal of Terramechanics, 44(4) :303 317, 2007.

[18] Ingo Scharfenbaum, Alessio Fratini, and Gunther Prokop. A Novel Method for the Development of an Idealised Active Roll Stabilisation System Model. 2013 IEEE International Conference on Systems, Man, and Cybernetics, pages 4499-4504, October 2013. 
[19] S. Zhou, S. Zhang, Semi-active control on leaf spring suspension based on smc, in : Control and Decision Conference, CDC 2010, Chinese, pp. $1462-1466$.

[20] F. Kou, Z. Fang, An experimental investigation into the design of vehicle fuzzy active suspension, in : IEEE International Conference Automation and Logistics, 2007, pp. $959-963$.

[21] B. Fleming. An overview of advances in automotive electronics [automotive electronics]. Vehicular Technology Magazine, IEEE, 9(1) :4-9, March 2014.

[22] D. Sampson, Active roll control of articulated heavy vehicles, Engineering department, Cambridge University Engineering Department, September 2000 .

[23] A. Miège, Active roll control of an experimental articulated vehicle, Ph.D. thesis, Cambridge University Engineering Department, October 2003.

[24] W. D. Jones. Easy ride : Bose corp. uses speaker technology to give cars adaptive suspension. IEEE Spectr., 42(5) :12-14, May 2005.

[25] P. He, Y. Wang, Y. Zhang, Y. Liu, and Y. Xu. Integrated control of semiactive suspension and vehicle dynamics control system. In Computer Ap- plication and System Modeling (ICCASM), 2010 International Conference on, volume 5, pages 63-68, 2010.

[26] B.L.J. Gysen, T.P.J. van der Sande, J.J.H. Paulides, and E.A. Lomonova. Efficiency of a regenerative direct-drive electromagnetic active suspension. Vehicular Technology, IEEE Transactions on, 60(4) :1384-1393, May 2011.

[27] Lifu Wang, Nong Zhang, and Haiping Du. Experimental investigation of a hydraulically interconnected suspension in vehicle dynamics and stability control. Technical report, SAE Technical Paper, 2012.

[28] Johan Berote. Dynamics and control of a tilting three wheeled vehicle. 2010 . 
[29] Y. Abbassi, Contribution à la modélisation et à la commande de la dynamique du véhicule, Ph.D. thesis, Université de Technologie BelfortMontbéliard / Université de Franche-Comté , 2007.

[30] J. Wong, Terramechanics and off-road Vehicles, Elsevier, 1989.

[31] Brad S., Tore H. and Anders R., Vehicle Dynamics Control and Controller Allocation for Rollover Prevention,in : IEEE International Conference on Control Application, October 2006, pp. 149 -154.

[32] H. Pacejka, Tyre and vehicle dynamics, Butterworth-Heinemann, 2002.

[33] N. Bouton, R. Lenain, B. Thuilot, J.-C. Fauroux, A rollover indicator based on the prediction of the load transfer in presence of sliding : application to an all terrain vehicle, in : IEEE International Conference Robotics and Automation, ICRA 2007, pp. 1158 -1163.

[34] R. Lenain, B. Thuilot, C. Cariou, P. Martinet, Mixed Kinematic and Dynamic Sideslip Angle Observer for Accurate Control of Fast Off-Road mobile Robots : Journal of Field Robotics Oct. 2009, Volume 27, Issue 2 . 